\title{
Efficient Enhanced Sleep Awake Scheduling Using Fuzzy Logic and Neural Networks : A Review \\ Mani $^{1}$, Er. Lovepreet Kaur ${ }^{2}$ \\ ${ }^{1}$ M. Tech Scholar, Guru Kashi University , Talwandi Sabo, Punjab, India \\ ${ }^{2}$ Assistant Professor, Guru Kashi University, Talwandi Sabo, Punjab, India
}

\begin{abstract}
Article Info

Volume 8 Issue 2

Page Number : $18-24$

Publication Issue :

March-April-2021

Article History

Accepted : 01 March 2021

Published : 08 March 2021

WSN is a distributed network that consists of great amount of sensor nodes and has the capacity of sensing, processing and transmits the partially processed and required data only. Sensor nodes have a tiny size, low cost but along with it the constraints of sensor node is they have limited memory, power source which is irreplaceable so power conservation should primarily focused by sensor network protocols. The proposed model was deals with environmental application where detection of forest fire is analyzed by taking parameters such as temperature, humidity, wind speed and time using fuzzy logic as by detecting earlier of fire in forest it helps to prevent huge loss of living organism, infrastructure and property. After detection the proposed MSA (Modified Sleep Awake) model work in prolonging lifetime of WSN in forest fire application using selective sleep awake approach. Cloud computing help to overcome the limitation of WSN such as limited storage, processing, power life processing. The resource allocation problem is the major problem for a group of cloud user requests. The scheduling algorithms are termed as NP completeness problems in which FIFO scheduling is used by the master node to distribute resources to the waiting tasks. The problem like fragmentation of resources, low utilization of the resources such as CPU utilization, network throughput, disk I/O rate. In this paper different papers are reviewed and further it is implemented in research paper.

Keywords : Cloud, FIFO, Internet, WSN, Distributed, Network
\end{abstract}

\section{INTRODUCTION}

Wireless Sensor Network: WSN is a distributed network that consists of great amount of sensor nodes and has the capacity of sensing, processing and transmit the partially processed and required data only. Sensor nodes have a tiny size, low cost but along with it the constraints of sensor node is they have limited memory, power source which is irreplaceable so power conservation should primarily focused by sensor network protocols[1] [2].The aim of wireless sensor network comprises reliability, accuracy, 
easiness of deployment and flexibility. Cloud Computing: Cloud computing is defined as computing paradigm for hosting and delivering services over the internet. Different academics, firms, IT companies and industry professionals defined cloud computing terms in many different ways. Cloud is large hub where easily virtualized resources (services, application, hardware, and platform) can easily accessible and usable. Merrill Lynch-“The idea of delivering personal (e g presentation, email, documents) and application of business productivity (e.g. accounting, sales force and other customer service) from centralized server [3].

Integration of WSN and Cloud: Integration of WSN with cloud computing: Cloud computing help to overcome the limitation of WSN such as limited storage, processing, power life processing. They exploit the cloud to share and process the sensory data as collected by WSN anytime and anywhere. . sensory data collected from different sensors is decomposed to the base station and is transferred to the cloud gateway, as Cloud provides capacity of storage through which internet users communicate with cloud and accessed the data from cloud from anywhere at any time. There are different applications where integration of sensor and cloud is widely used such as: For disaster detection environment monitoring, health care monitoring, transportation and vehicle monitoring, agriculture and irrigation control and so on. For different types of analysis the integration of WSN and cloud makes more interesting to the users. For industry and other organization instead of storing data in their private system cloud help to provide storage capacity so that they can store and access the data from anywhere as response time of cloud is greater than other system.

\section{LITERATURE SURVEY}

In this paper I have studied the different papers to review my research topic. I have studied different authors papers .each have followed the different techniques and methods.

Rashi Srivastava et.al.[2018] have been proposed the integration of Wireless Sensor Network (WSN) with Cloud is becoming popular in most of the industry and academics. The proposed model was deals with environmental application where detection of forest fire is analyzed by taking parameters such as temperature, humidity, wind speed and time using fuzzy logic as by detecting earlier of fire in forest it helps to prevent huge loss of living organism, infrastructure and property. After detection the proposed MSA (Modified Sleep Awake) model work in prolonging lifetime of WSN in forest fire application using selective sleep awake approach.[28]

Mehmet Şimşek et.al. [2018] have been studied WSN is a technology that provides distributed data collection. However, these networks have some limitations. In this study, they proposed an application specific method to wake up and sleep nodes in WSNs. In traditional strategy, the nodes in the cluster sense data and send it to the Cluster Head $(\mathrm{CH})$, if $\mathrm{CHs}$ detect redundancy of some data; they remove the duplication and send it to the base station. This is causing energy loss. Our method puts some nodes to sleep state if there are similar data in a certain period. By this way, the life of the network is extended.[20]

Ms. Raksha Gound et.al.[2017] have been studied Existing sleep scheduling algorithms cause an extremely unbalanced energy usage, and due to this, some sensors reduce the overall network's lifetime. The proposed system uses a sensor node to which event occurs and transmits data to base station and its neighbor nodes in a particular area of WSN and which improves energy efficiency. The sleep scheduling based on the location of node minimizes the power consumption of WSN. The simulation result shows that the resulting representative data 
achieved using the proposed algorithm have better throughput and average energy consumption than those achieved using CLSS1 and CLSS2 algorithms.[21]

Karthihadevi. M et.al.[2017] have been studied a Wireless Sensor Network (WSN) is a distributed network with a large number of distributed, selfdirected, low powered, tiny devices called sensor nodes. To extend the lifetime of wireless sensor network, the design of Energy-Efficient scheduling algorithm is an important factor. In order to optimize the energy of sensors different mechanisms can be used and they have a great impact on prolonging the network lifetime. Various algorithms have been propounded to minimize the energy by scheduling sensor nodes activity. In this paper various sensor nodes sleep scheduling algorithms are compared and analyzed.[17]

Shubhra Saxena et.al.[2018] have studied Cloud computing (CC) is rising rapidly; an expansive number of clients are pulled in towards cloud administrations for more fulfillments. Distributed computing is most recent developing innovation for expansive scale dispersed processing and parallel registering. Better load adjusting calculation in cloud framework builds the execution and assets use by progressively dispersing work stack among different hubs in the framework. Virtual machine (VM) is an execution unit that goes about as an establishment for distributed computing innovation [33]

Renu Choudhary et.al.[2018] have studied Cloud computing is an interesting era of research, where motivation is to find out the best outcome and productive data security and sharing approach. Load balancing in public impair by way of division of cloud just right geographical position. Load balancing is frequently a strategy of controlling the visitors in a cloud atmosphere. Cloud requests hunt for assets for performance. The resources are quite often storage, processing, bandwidth, and many others. Allocation these belongings efficaciously to the entire competing jobs are named as load balancing. This paper will provide a comprehensive survey of cloud load balancing techniques.[29]

Priyam Tyagi et.al.[2018] have studied - Cloud Computing is a computing interpretation which provides convenient way to access resources and in which data can be stored on paid basics. Load Balancing is one of the methods in Cloud Computing which helps in balancing loads as it increase the throughput and minimize the response time. It distributes loads uniformly on nodules and increase overall performance in the system. The aim of Load balancing is to allocate resources and guarantees user satisfaction. In this paper I explore two of the Cloud computing Algorithms to overcome load balancing in it.[23]

A Arul Prakash et.al.[2018] have studied . Load balancing has turned out to be significant for productive execution in appropriated conditions. Cloud computing is a developing innovation requesting more administrations and better outcomes. This paper examines Cloud computing alongside investigate challenges in load balancing. Load balancing has been a significant issue for Cloud computing condition. The point of this examination is to peep in different load balancing calculations to address its difficulties in assortment of cloud condition. This examination gives a point of view perspective of the most recent methodologies in load balancing that will unquestionably help the future scientists in this field.[1]

Cheng Fang Zhen et.al.[2014] have been studied Both energy-saving and synchronization issues are the paramount concern in wireless sensor networks (WSNs). In this paper we propose a simple and efficient WSN node design based on acoustic positioning applications and present an on-demand sleep/wake scheduling synchronization protocol. The 
on-demand synchronization protocols are implemented in sensor nodes and evaluated in a testbed. Analysis and simulation were performed that the proposed protocol has significantly reduced the energy consumption. It is also demonstrated by experiments that the platform is accurate and effective.[6]

Saeed javanmardi et al. [2014] have been proposed the aid of genetic algorithm and fuzzy theory, present a hybrid job scheduling approach, which consider the load balancing of the system and reduces total execution time and execution cost. The main goal of the research is to assign the jobs to the resources with considering the VM MIPS and time-span of jobs. The new algorithm assigns the jobs to the resources with considering the job length and resources capacities. Evaluate the performance of the approach with some famous cloud scheduling models. The result of the experiments shows the efficiency of the proposed approach in term of execution time, execution cost and average degree of imbalance [31].

Rajveer Kaur et al. [2014] have been proposed the internet based computing while software, information and shared resources are provided to devices and computers on demand, like electricity grid. With the fusion of network technology and traditional computing technology such as distributed computing parallel computing, grid computing a cloud computing product is formed. Here in this work, genetic algorithm is enhanced using new fitness function based on mean and grand mean values. This optimization can be implemented on both ends, for job scheduling and resource scheduling. This will schedule the whole process and optimize as much as possible. The results analysis also proves the cloud system's increased efficiency for task scheduling.[27]

Dr. Amit Agarwal et.al. [2014] have been suggested that the cloud computing is an emerging technology in distributed computing which facilitates pay per model as per user demand and requirement. Cloud consist of a collection of virtual machine which includes both computational and storage facility. A good scheduler adapts its scheduling strategy according to the changing environment and the type of task. In this research paper presented a Generalized Priority algorithm for efficient execution of task and comparison with FCFS and Round Robin Scheduling. Algorithm should be tested in cloud Sim toolkit and result shows that it gives better performance compared to other traditional scheduling algorithm.[23]

Shilpa V Pius et al.[2014] have been discussed that the cloud computing is one of the most interesting research areas having great impact on technological development. This paper focuses on survey of various load balancing methods. During the recent years many load balancing approaches are proposed in the area of cloud computing. For further researches, understanding of these approaches is essential.[32]

Hitesh A. Ravani et al. [2013] have been discusses that Resource Scheduling is the process of mapping tasks to available resources on the basis of tasks characteristics and requirements. The received tasks are group on the basis of data and resources. Main aim of this paper is to analyze the various scheduling algorithm and manage the resources which are precisely available at certain fixed times and for fifed intervals of time. Find the optimizes scheduling algorithm for resource so the cloud provider get benefits in term of efficient resource management which provide more resources to allocate without postponing or declining any user requests. Cloud users also get benefits in term of their monetary gains at each front [13].

Florin Pop et al. [2013] have been evolutionary computing offers different methods to solve NP-hard problems, finding a near-optimal solution. Task scheduling is a composite problem for large 
environments like Clouds. Genetic algorithms are a superior method to find a solution for this problem considering multi-criteria constrains. On the other hand, a good performance to ensure the QoS is to use the reputation of resources offered. This aspect is very important for service providers because represents a ranking method for them. The characters is considered in the selection phase of genetic algorithm as evolutionary criteria for the algorithm and evaluate the proposed solution considering load-balancing as a way to measure the optimization impact for providers and maxspan as a metric for user performance [10].

Tarun Goyal et al.[2013] have been represents that cloud computing is a paradigm in which IT (information technology) application provide as a service. Cloud computing allows users to utilize the computation, storage, data and services from around the world in commercialize manner. In cloud environment, scheduling is the major issue. Scheduling is responsible efficient utilization of the resources. In this paper, a Scheduling model based on minimum network delay using Suffrage Heuristic coupled with Genetic algorithms for scheduling sets of independent jobs algorithm is proposed, the objective is to minimize the make span.[35]

P. Mohamed Shameem et al.[2013] have been proposed that cloud computing offers a variety of dynamic flexible resources to expedite the processing of large scale tasks in pay-by-use manner to public. Moreover, the method is required to allocate dynamic workloads equally to all the nodes across cloud network. Proper load balancing technique helps in implementing fail-over, avoiding bottleneck problems, enabling scalability, optimizing resource allocation etc. This paper presents a survey about dynamic load balancing strategies directed on cloud data storage on workloads.[22]

Jianfeng Zhao et al. [2011] have been proposed a virtual resources scheduling model and solved it by advanced Non-dominated Sorting Genetic Algorithm
II (NSGA II). This model was evaluated by balance load, virtual resources and physical resources were abstracted a lot of nodes with attributes based on analyzing the flow of virtual resources scheduling. In experiment, verified the correctness of this model. Comparing with Random algorithm, Static algorithm and Rank algorithm by a lot of experiments, at least 1.06 and at most 40.25 speed-up of balance degree can be obtained by NSGA II [37].

Lucio Agostinho [2011] have been proposed paper discussed about the cloud computing the allocation and scheduling of multiple virtual resources, such as virtual machines (VMs), are still a challenge. The optimization of these processes brings the advantage of improving the energy savings and load balancing in large datacenters. The main contribution of this work is an inter-domain allocation algorithm that takes into account the capacity of the links connecting the domains in order to avoid quality of service degradation for VMs allocated on partner domains. Architecture to replicate federated clouds is also a contribution of this paper [18].

\section{III.CONCLUSION \& FUTURE WORK}

Cloud computing is defined as computing paradigm for hosting and delivering services over the internet. Cloud computing help to overcome the limitation of WSN such as limited storage, processing, power life processing. They exploit the cloud to share and process the sensory data as collected by WSN anytime and anywhere. . sensory data collected from different sensors is decomposed to the base station and is transferred to the cloud gateway, as Cloud provides capacity of storage through which internet users communicate with cloud and accessed the data from cloud from anywhere at any time. In the future it is implemented with different toolboxes and to resolve the security problems on different cloud areas. 


\section{REFERENCES}

[1]. A Arul Prakash, V Arul, A Jagannathan “A Look at of Efficient and more Suitable Load Balancing Algorithms in Cloud Computing" International Journal of Engineering Research in Computer Science and Engineering (IJERCSE) Vol 5, Issue 4, April 2018.

[2]. A. d. Costanzo, M. D. d. Assunção, R. Buyya. "Harnessing Cloud Technologies for a Virtualized," Distributed Computing Infrastructure, vol. 13, pp. 24-33, Octobor 2009.

[3]. Andrew J. Younge, Gregor von Laszewski, Lizhe Wang, Sonia Lopez-Alarcon, warren Carithers, "Efficient Resource Management for Cloud Computing Environments", IEEE, 2010, pp.

[4]. Anthony T.Velte, Toby J.Velte, Robert Elsenpeter, "Cloud Computing A Practical Approach", TATA McGRAW-HILL Edition 2010.

[5]. C. Shi, Z. Yan, Z. Shi, L. Zhang. "A fast multiobjective evolutionary algorithm based on a tree structure," Applied Soft Computing, vol. 10,pp. 468-480, Feburary 2010.

[6]. ChengFang Zhen et.al. "Energy-Efficient Sleep/Wake Scheduling for Acoustic Localization Wireless Sensor Network Node" International Journal of Distributed Sensor Networks 2014.

[7]. D.T. Pham, A. Ghanbarzadeh, E. Koc, S. Otri, S. Rahim, M. Zaidi, "The Bees Algorithm-A Novel Tool for Complex Optimization Problem", Cardiff CF243AA UK, 2012

[8]. Dr. Amrit Agarwal, Saloni Jain, "Efficient Optimal Algorithm of Task Scheduling in Cloud Computing Environment", International Journal of Computer Trends and Technology(IJCTT)-Volume 9, 7 march 2014.

[9]. Er. Shimpy, Mr. Jagandeep Sidhu, "Different Scheduling Algorithms In Different Cloud Environment", International Journal of
Advanced Research in Computer and Communication Engineering, Vol. 3, Issue 9, September 2014, ISSN: 2278-1021

[10]. Florin Pop, Valentin cristea, Nik Bbbessis, Stelious Sotiriadis, "Reputation guided Genetic Scheduling Algorithm for Independent Tasks in Inter-Clouds Environments", International Conference on Advanced Information Networking and Applications Workshops, 2013, pp. 772-776.

[11]. G. Tian, D. Meng, J. Zhan. "Reliable Resource Provision Policy for Cloud Computing," Chinese Journal of computer, vol. 33, pp. 18591872, Octobor 2010.

[12]. Gaochao Xu, Junjie Pang and Xiaodong Fu, “ A Load Balancing Model Based on Cloud Partitioning for the Public Cloud," Tsinghua Science and Technology, ISSN: 1007-0214, Vol. 18, No. 1, Feb. 2013, pp. 34-39.

[13]. Hitesh A Ravani, Hitesh A Bheda, "Genetic Algorithm Based resource Scheduling Technique in Cloud Computing”, International Journal in Advance Research of Computer Science and Management Studies, Volume 1, Issue 7, December 2013, ISSN 2321-7782.

[14]. Jaspreet Kaur, "Comparison of Load Balancing Algorithms in Cloud," International Journal of Engineering and Applications(IJERA), ISSN: 2248-9622, Vol. 2, Issue 3, May-Jun 2012,pp.1169-1173.

[15]. Jianfeng Zhao, Wenhua Zeng, Min Liu, Guangming Li" Multi-objective Optimization Model of Virtual Resources Scheduling Under Cloud Computing and It's Solution" International Conference on Cloud and Service Computing,2011.

[16]. Kai Zhu, Huaguang Song, Lijing Liu, Jinzhu Gao, Guojian Cheng, " Hybrid Genetic Algorithm for Cloud Computing Applications," IEEE Asia-Pacific services Computing Conference 2011, DOI 10.1109/APSCC.2011.66 
[17]. Karthihadevi. M et.al. "Sleep Scheduling Strategies In Wireless Sensor Network" Advances In Natural And Applied Sciences, 2017 May 11(7): pages 635-641.

[18]. Lucio Agostinho, Guilherme Feliciano, Leonardo Olivi, Eleri Cardozo" A Bio-inspired Approach to Provisioning of Virtual Resources in Federated Clouds" IEEE Ninth International Conference on Dependable, Autonomic and Secure Computing,2011.

\section{Cite this article as :}

Mani, Er. Lovepreet Kaur, "Efficient Enhanced Sleep Awake Scheduling Using Fuzzy Logic and Neural Networks : A Review", International Journal of Scientific Research in Science, Engineering and Technology (IJSRSET), Online ISSN : 2394-4099, Print ISSN : 2395-1990, Volume 8 Issue 2, pp. 18-24, March-April 2021. Available at doi : https://doi.org/10.32628/IJSRSET21828

Journal URL : https://ijsrset.com/IJSRSET21828 